International Mathematical Forum, 1, 2006, no. 17, 823-832

\title{
Two Step Method for Fuzzy Differential Equations
}

\author{
T. Allahviranloo ${ }^{1}$, N. Ahmady, E. Ahmady \\ Department of Mathematics \\ Science and Research Branch Islamic \\ Azad University, Tehran, Iran
}

\begin{abstract}
In this paper a numerical algorithm for solving 'fuzzy ordinary differential equations' is considered. A scheme based on the two step method in detail is discussed. The algorithm is illustrated by solving fuzzy Cauchy problem.
\end{abstract}

Keywords: Fuzzy Differential Equation, Two Step Method, Fuzzy Cauchy Problem

\section{Introduction}

The concept of fuzzy derivative was first introduced by S.L. Chang, L.A. Zadeh in [3] it was followed up by D.Dubois, H. Prade in [4], who defined and used the extension principle. The fuzzy differential equation and the fuzzy initial value problem were regularly treated by O. Kaleva in $[5,6]$ and by S. Seikkala in $[7], \ldots$ The numerical method for solving fuzzy differential equations is introduced in $[1,2,8]$ by the standard Euler method. This paper organize as follows:

In section 2, we being some basic definitions and results which will be used later. In section 3 we define the problem, this is a fuzzy Cauchy problem whose numerical solution is the main interest of this work. The numerically solving fuzzy differential equation by two step Method is discussed in this section. The proposed algorithm is illustrated by solving an example in section 4 and conclusions are drawn in section 5 .

\footnotetext{
${ }^{1}$ tofigh@allahviranloo.com
} 


\section{Preliminaries}

\section{1 notation and definition}

Definition 2.1 An m-step multi step method for solving the initial-value problem is one whose difference equation for finding the approximation $y\left(t_{i+1}\right)$ at the mesh point $t_{i+1}$ can be represented by the following equation, where $m$ is an integer greater than 1:

$$
\begin{aligned}
y\left(t_{i+1}\right) & =a_{m-1} y\left(t_{i}\right)+a_{m-2} y\left(t_{i-1}\right)+\ldots+a_{0} y\left(t_{i+1-m}\right) \\
+h\left\{b_{m} f\left(t_{i+1}, y_{i+1}\right)\right. & \left.+b_{m-1} f\left(t_{i}, y_{i}\right)+\ldots+b_{0} f\left(t_{i+1-m}, y_{i+1-m}\right)\right\}
\end{aligned}
$$

for $i=m-1, m, \ldots, N-1$, where $a=t_{0} \leq t_{1} \leq \ldots \leq t_{N}=b$ and $h=\frac{(b-a)}{N}=$ $t_{i+1}-t_{i}$, the $a_{0}, a_{1}, \ldots, a_{m-1}$ and $b_{0}, b_{1}, \ldots, b_{m}$ are constants, and the starting values

$$
y_{0}=\alpha_{0}, \quad y_{1}=\alpha_{1}, \quad y_{2}=\alpha_{2}, \ldots, \quad y_{m-1}=\alpha_{m-1}
$$

are specified.

When $b_{m}=0$, the method is called explicit, since Eq.2.1 then gives $y_{i+1}$ explicit in terms of previously determined values. When $b_{m} \neq 0$, the method is called implicit, since $y_{i+1}$ occurs on both sides of Eq.(2.1) and is specified only implicit.

Adams-Bashforth two-step method is as follows

$$
\begin{gathered}
y_{0}=\alpha_{0}, \quad y_{1}=\alpha_{1}, \\
y_{i+1}=y_{i}+\frac{h}{2}\left[3 f\left(t_{i}, y_{i}\right)-f\left(t_{i-1}, y_{i-1}\right)\right],
\end{gathered}
$$

where $i=1,2, \ldots, N-1$.

Let $E$ be the set of all upper semicontinuous normal convex fuzzy numbers with bounded $\alpha$-level intervals. It means that if $v \in E$ then the $\alpha$-level set

$$
[v]^{\alpha}=\{s \mid v(s) \geq \alpha\}, \quad 0<\alpha \leq 1
$$

is a closed bounded interval which is denoted by

$$
[v]^{\alpha}=\left[v_{1}(\alpha), v_{2}(\alpha)\right] .
$$

Let $I$ be a real interval. A mapping $y: I \rightarrow E$ is called a fuzzy process and its $\alpha$-level set is denoted by

$$
[y(t)]^{\alpha}=\left[y_{1}(t ; \alpha), y_{2}(t ; \alpha)\right], \quad t \in I, \quad \alpha \in(0,1] .
$$

The triangular fuzzy numbers are those fuzzy sets in $E$ which are characterized by an ordered triple $\left(x_{l}, x_{c}, x_{r}\right) \in R^{3}$ with $x_{l} \leq x_{c} \leq x_{r}$ such that $[U]^{0}=\left[x_{l}, x_{r}\right]$ 
and $[U]^{1}=\left\{x_{c}\right\}$ for then

$$
[U]^{\alpha}=\left[x_{c}-(1-\alpha)\left(x_{c}-x_{l}\right), x_{c}+(1-\alpha)\left(x_{r}-x_{c}\right)\right]
$$

for any $\alpha \in I$.

Definition 2.2 The supremum metric $d_{\infty}$ on $E^{n}$ is defined by

$$
d_{\infty}(U, V)=\sup \left\{d_{H}\left([U]^{\alpha},[V]^{\alpha}\right): \alpha \in I\right\}
$$

and $\left(E^{n}, d_{\infty}\right)$ is a complete metric space.

Definition 2.3 A mapping $F: T \longrightarrow E^{n}$ is Hukuhara differentiable at $t_{0} \in$ $T \subseteq R$ if for some $h_{0}>0$ the Hukuhara differences

$$
F\left(t_{0}+\Delta t\right) \sim_{h} F\left(t_{0}\right), F\left(t_{0}\right) \sim_{h} F\left(t_{0}-\Delta t\right)
$$

exist in $E^{n}$ for all $0<\Delta t<h_{0}$ and if there exists an $F^{\prime}\left(t_{0}\right) \in E^{n}$ such that

$$
\left.\lim _{\Delta t \rightarrow 0+} d_{\infty}\left(F\left(t_{0}+\Delta t\right) \sim_{h} F\left(t_{0}\right)\right) / \Delta t, F^{\prime}\left(t_{0}\right)\right)=0
$$

and

$$
\left.\lim _{\Delta t \rightarrow 0+} d_{\infty}\left(F\left(t_{0}\right) \sim_{h} F\left(t_{0}-\Delta t\right)\right) / \Delta t, F^{\prime}\left(t_{0}\right)\right)=0
$$

the fuzzy set $F^{\prime}\left(t_{0}\right)$ is called the Hukuhara derivative of $F$ at $t_{0}$.

Recall that $U \sim_{h} V=W \in E^{n}$ is define on level sets, that $[U]^{\alpha} \sim_{h}[V]^{\alpha}=$ $[W]^{\alpha}$ for all $\alpha \in I$. In view of the definition of the metric $d_{\infty}$, all the level set mappings $[F(.)]^{\alpha}$ are Hukuhara differentiable at $t_{0}$ with Hukuhara derivatives $\left[F^{\prime}\left(t_{0}\right)\right]^{\alpha}$ for each $\alpha \in I$ when $F: T \longrightarrow E^{n}$ is Hukuhara differentiable at $t_{0}$ with Hukuhara derivative $F^{\prime}\left(t_{0}\right)$.

Definition 2.4 The fuzzy integral

is defined by

$$
\int_{a}^{b} y(t) d t \quad a, b \in I
$$

$$
\left[\int_{a}^{b} y(t) d t\right]^{\alpha}=\left[\int_{a}^{b} \underline{y}^{\alpha}(t) d t, \int_{a}^{b} \bar{y}^{\alpha}(t) d t\right]
$$

provided that the Lebesgue integrals on the right exist.

Remark 2.1 If $F: T \longrightarrow E^{n}$ is Hukuhara differentiable and its Hukuhara derivative $F^{\prime}$ is integrable over $[0,1]$, then

$$
F(t)=F\left(t_{0}\right)+\int_{t_{0}}^{t} F^{\prime}(s) d s
$$

for all $0 \leq t_{0} \leq t \leq 1$. 


\subsection{Interpolation for fuzzy number}

The interpolation polynomial can be written level setwise as

$$
[f(t)]^{\alpha}=\left\{y \in R: y=P_{X}(t), x \in\left[u_{i}\right]^{\alpha}, i=1,2, \ldots, n\right\}, \text { for } 0 \leq \alpha \leq 1 .
$$

When the data $u_{i}$ presents as triangular fuzzy numbers, value of the interpolation polynomial are also triangular fuzzy numbers. Then $f(t)$ has particularly simple form that is well suited to computation. Denote by $\ell_{i}(t), i=1,2, \ldots, n$, the polynomials

$$
\ell_{i}(t)=\prod_{j \neq i}^{n} \frac{t-t_{j}}{t_{i}-t_{j}}
$$

Theorem 2.1 Let $\left(t_{i}, u_{i}\right), i=1,2, \ldots, n$ be the observed data and suppose that each of the $u_{i}=\left(u_{i}^{l}, u_{i}^{c}, u_{i}^{r}\right)$ is an element of $E$. Then for each $t \in\left[t_{0}, t_{n}\right]$ , $f(t)=\left(f^{l}(t), f^{c}(t), f^{r}(t)\right)$ is in $E$ and $f^{l}(t)=\sum_{\ell_{i}(t) \geq 0} \ell_{i}(t) u_{i}^{l}+\sum_{\ell_{i}(t)<0} \ell_{i}(t) u_{i}^{r}$

$f^{c}(t)=\sum \ell_{i}(t) u_{i}^{c}$

$f^{r}(t)=\sum_{\ell_{i}(t) \geq 0} \ell_{i}(t) u_{i}^{r}+\sum_{\ell_{i}(t)<0} \ell_{i}(t) u_{i}^{l}$.

proof. see[12]

\section{A fuzzy Cauchy problem}

Consider a first order fuzzy differential equation by $y^{\prime}=f(t, y)$ where $y$ is a fuzzy function of $t, f(t, y)$ is a fuzzy function of the crisp variable $t$ and the fuzzy variable $y$, and $y^{\prime}$ is the fuzzy Hukuhara derivative of $y$. If an initial value $\widetilde{y}(a)=\widetilde{\alpha}_{0}$ is given, we obtain a fuzzy cauchy problem of first order:

$$
\left\{\begin{array}{l}
y^{\prime}(t)=f(t, y(t)) ; \quad a \leq t \leq b \\
\widetilde{y}(a)=\widetilde{\alpha}_{0}
\end{array}\right.
$$

Sufficient conditions for the existence of a unique solution to Eq.(3.3) are that $f$ is continuous and that Lipschitz condition

$$
d_{\infty}(f(t, x), f(t, y)) \leq L d_{\infty}(x, y), \quad L>0
$$

is fulfilled. 


\subsection{Two Step Method}

For solve fuzzy initial value problem $y^{\prime}(t)=f(t, y(t))$ by two step method, let the fuzzy initial value be $y\left(t_{i}\right), y\left(t_{i-1}\right)$, i.e

$$
\widetilde{f}\left(t_{i-1}, y\left(t_{i-1}\right)\right), \widetilde{f}\left(t_{i}, y\left(t_{i}\right)\right)
$$

are defined. Which are triangular fuzzy numbers and we show them with :

$$
\begin{gathered}
\left(f^{l}\left(t_{i-1}, y\left(t_{i-1}\right)\right), f^{c}\left(t_{i-1}, y\left(t_{i-1}\right)\right), f^{r}\left(t_{i-1}, y\left(t_{i-1}\right)\right)\right), \\
\left(f^{l}\left(t_{i}, y\left(t_{i}\right)\right), f^{c}\left(t_{i}, y\left(t_{i}\right)\right), f^{r}\left(t_{i}, y\left(t_{i}\right)\right)\right) .
\end{gathered}
$$

By remark (2.1) we have :

$$
\widetilde{y}\left(t_{i+1}\right)=\widetilde{y}\left(t_{i}\right)+\int_{t_{i}}^{t_{i+1}} \widetilde{f}(t, y(t)) d t
$$

Because $\widetilde{y}(t)$ is unknown, we use fuzzy interpolation on fuzzy numbers $\widetilde{f}\left(t_{i-1}, y\left(t_{i-1}\right)\right), \widetilde{f}\left(t_{i}, y\left(t_{i}\right)\right)$, hence by theorem (2.1) we obtain :

$f^{l}(t, y(t))=\sum_{j=i-1 \ell_{j}(t) \geq 0}^{i} \ell_{j}(t) f^{l}\left(t_{j}, y\left(t_{j}\right)\right)+\sum_{j=i-1 \ell_{j}(t)<0}^{i} \ell_{j}(t) f^{r}\left(t_{j}, y\left(t_{j}\right)\right)$

$f^{c}(t, y(t))=\sum_{j=i-1}^{i} \ell_{j}(t) f^{c}\left(t_{j}, y\left(t_{j}\right)\right)$

$f^{r}(t, y(t))=\sum_{j=i-1 \ell_{j}(t) \geq 0}^{i} \ell_{j}(t) f^{r}\left(t_{j}, y\left(t_{j}\right)\right)+\sum_{j=i-1 \ell_{j}(t)<0}^{i} \ell_{j}(t) f^{l}\left(t_{j}, y\left(t_{j}\right)\right)$

for $t_{i} \leq t \leq t_{i+1}$ we have :

$\ell_{i-1}(t)=\frac{\left(t-t_{i}\right)}{\left(t_{i-1}-t_{i}\right)} \leq 0, \ell_{i}(t)=\frac{\left(t-t_{i-1}\right)}{\left(t_{i}-t_{i-1}\right)} \geq 0$

therefore :

$$
\begin{aligned}
& f^{l}(t, y(t))=\ell_{i-1}(t) f^{r}\left(t_{i-1}, y\left(t_{i-1}\right)\right)+\ell_{i}(t) f^{l}\left(t_{i}, y\left(t_{i}\right)\right) \\
& f^{c}(t, y(t))=\ell_{i-1}(t) f^{c}\left(t_{i-1}, y\left(t_{i-1}\right)\right)+\ell_{i}(t) f^{c}\left(t_{i}, y\left(t_{i}\right)\right) \\
& f^{r}(t, y(t))=\ell_{i-1}(t) f^{l}\left(t_{i-1}, y\left(t_{i-1}\right)\right)+\ell_{i}(t) f^{r}\left(t_{i}, y\left(t_{i}\right)\right)
\end{aligned}
$$

By using (2.2) and (3.4) we have :

$$
\widetilde{y}^{\alpha}\left(t_{i+1}\right)=\left[\underline{y}^{\alpha}\left(t_{i+1}\right), \bar{y}^{\alpha}\left(t_{i+1}\right)\right]
$$

with

$$
\underline{y}^{\alpha}\left(t_{i+1}\right)=\underline{y}^{\alpha}\left(t_{i}\right)+\int_{t_{i}}^{t_{i+1}} \alpha f^{c}(t, y(t))+(1-\alpha) f^{l}(t, y(t)) d t
$$


and

$$
\bar{y}^{\alpha}\left(t_{i+1}\right)=\bar{y}^{\alpha}\left(t_{i}\right)+\int_{t_{i}}^{t_{i+1}} \alpha f^{c}(t, y(t))+(1-\alpha) f^{r}(t, y(t)) d t
$$

If we situate (3.5), (3.6) in (3.8) and (3.6), (3.7) in (3.9) we will have :

$$
\begin{gathered}
\underline{y}^{\alpha}\left(t_{i+1}\right)=\underline{y}^{\alpha}\left(t_{i}\right)+\int_{t_{i}}^{t_{i+1}}\left\{\alpha\left(\ell_{i-1}(t) f^{c}\left(t_{i-1}, y\left(t_{i-1}\right)\right)+\ell_{i}(t) f^{c}\left(t_{i}, y\left(t_{i}\right)\right)\right)+\right. \\
\left.(1-\alpha)\left(\ell_{i-1}(t) f^{r}\left(t_{i-1}, y\left(t_{i-1}\right)\right)+\ell_{i}(t) f^{l}\left(t_{i}, y\left(t_{i}\right)\right)\right)\right\} d t \\
\bar{y}^{\alpha}\left(t_{i+1}\right)=\bar{y}^{\alpha}\left(t_{i}\right)+\int_{t_{i}}^{t_{i+1}}\left\{\alpha\left(\ell_{i-1}(t) f^{c}\left(t_{i-1}, y\left(t_{i-1}\right)\right)+\ell_{i}(t) f^{c}\left(t_{i}, y\left(t_{i}\right)\right)\right)+\right. \\
\left.(1-\alpha)\left(\ell_{i-1}(t) f^{l}\left(t_{i-1}, y\left(t_{i-1}\right)\right)+\ell_{i}(t) f^{r}\left(t_{i}, y\left(t_{i}\right)\right)\right)\right\} d t
\end{gathered}
$$

By integration we have :

$$
\begin{aligned}
& \underline{y}^{\alpha}\left(t_{i+1}\right)=\underline{y}^{\alpha}\left(t_{i}\right)-\left(\frac{h}{2}\right)\left(\alpha f^{c}\left(t_{i-1}, y\left(t_{i-1}\right)\right)+(1-\alpha) f^{r}\left(t_{i-1}, y\left(t_{i-1}\right)\right)\right) \\
&+\left(\frac{3 h}{2}\right)\left(\alpha f^{c}\left(t_{i}, y\left(t_{i}\right)\right)+(1-\alpha) f^{l}\left(t_{i}, y\left(t_{i}\right)\right)\right) \\
& \bar{y}^{\alpha}\left(t_{i+1}\right)= \bar{y}^{\alpha}\left(t_{i}\right)-\left(\frac{h}{2}\right)\left(\alpha f^{c}\left(t_{i-1}, y\left(t_{i-1}\right)\right)+(1-\alpha) f^{l}\left(t_{i-1}, y\left(t_{i-1}\right)\right)\right) \\
&\left.+\left(\frac{3 h}{2}\right)\left(\alpha f^{c}\left(t_{i}, y\left(t_{i}\right)\right)\right)+(1-\alpha) f^{r}\left(t_{i}, y\left(t_{i}\right)\right)\right)
\end{aligned}
$$

thus:

$$
\begin{aligned}
& \underline{y}^{\alpha}\left(t_{i+1}\right)=\underline{y}^{\alpha}\left(t_{i}\right)-\left(\frac{h}{2}\right) \bar{f}^{\alpha}\left(t_{i-1}, y\left(t_{i-1}\right)\right)+\left(\frac{3 h}{2}\right) \underline{f}^{\alpha}\left(t_{i}, y\left(t_{i}\right)\right) \\
& \bar{y}^{\alpha}\left(t_{i+1}\right)=\bar{y}^{\alpha}\left(t_{i}\right)-\left(\frac{h}{2}\right) \underline{f}^{\alpha}\left(t_{i-1}, y\left(t_{i-1}\right)\right)+\left(\frac{3 h}{2}\right) \bar{f}^{\alpha}\left(t_{i}, y\left(t_{i}\right)\right)
\end{aligned}
$$

By (3.10) and (3.11) we have :

$$
\left\{\begin{aligned}
\underline{y}^{\alpha}\left(t_{i+1}\right) & =y^{\alpha}\left(t_{i}\right)-\left(\frac{h}{2}\right) \bar{f}^{\alpha}\left(t_{i-1}, y\left(t_{i-1}\right)\right)+\left(\frac{3 h}{2}\right) f^{\alpha}\left(t_{i}, y\left(t_{i}\right)\right) \\
\bar{y}^{\alpha}\left(t_{i+1}\right) & =\bar{y}^{\alpha}\left(t_{i}\right)-\left(\frac{h}{2}\right) \underline{f}^{\alpha}\left(t_{i-1}, y\left(t_{i-1}\right)\right)+\left(\frac{3 h}{2}\right) \bar{f}^{\alpha}\left(t_{i}, y\left(t_{i}\right)\right) \\
y^{\alpha}\left(t_{i-1}\right) & =\alpha_{0}, y^{\alpha}\left(t_{i}\right)=\alpha_{1} \\
\bar{y}^{\alpha}\left(t_{i-1}\right) & =\alpha_{2}, \bar{y}^{\alpha}\left(t_{i}\right)=\alpha_{3}
\end{aligned}\right.
$$

To integrate the system given Eq.(3.12) from $t_{0}$ a prefixed $T>t_{0}$, we replace the interval $\left[t_{0}, T\right]$ by a set of discrete equally spaced grid points $t_{0}<t_{1}<t_{2}<$ $\ldots<t_{N}=T$ at which the exact solution $[\underline{Y}(t, \alpha), \bar{Y}(t, \alpha)]$ is approximated by some $[\underline{y}(t, \alpha), \bar{y}(t, \alpha)]$. The exact and approximate solutions at $t_{n}, 0 \leq$ $n \leq N$ are denoted by $Y_{n}(\alpha)=\left[\underline{Y}_{n}(\alpha), \bar{Y}_{n}(\alpha)\right]$ and $y_{n}(\alpha)=\left[\underline{y}_{n}(\alpha), \bar{y}_{n}(\alpha)\right]$, respectively. The grid points at which the solution is calculated are $t_{n}=$ 
$t_{0}+n h, \quad h=\left(T-t_{0}\right) / N ; 1 \leq n \leq N$.

from Eq.(3.12), the polygon curves

$$
\begin{aligned}
& \underline{y}(t, h, \alpha)=\left\{\left[t_{0}, \underline{y}_{0}(\alpha)\right],\left[t_{1}, \underline{y}_{1}(\alpha)\right], \ldots,\left[t_{N}, \underline{y}_{N}(\alpha)\right]\right\} \\
& \bar{y}(t, h, \alpha)=\left\{\left[t_{0}, \bar{y}_{0}(\alpha)\right],\left[t_{1}, \bar{y}_{1}(\alpha)\right], \ldots,\left[t_{N}, \bar{y}_{N}(\alpha)\right]\right\}
\end{aligned}
$$

are the two step approximates to $\underline{Y}(t, \alpha)$ and $\bar{Y}(t, \alpha)$ respectively over the interval $t_{0} \leq t \leq t_{N}$. The following lemma will be applied to show convergence of these approximates, i.e.,

$$
\lim _{h \rightarrow 0} \underline{y}(t, h, \alpha)=\underline{Y}(t, \alpha), \quad \lim _{h \rightarrow 0} \bar{y}(t, h, \alpha)=\bar{Y}(t, \alpha)
$$

Lemma 3.1 Let a sequence of numbers $\left\{w_{n}\right\}_{n=0}^{N}$ satisfy :

$$
\left|w_{n+1}\right| \leq A\left|w_{n}\right|+B\left|w_{n-1}\right|+C, \quad 0 \leq n \leq N-1
$$

for some given positive constants $A$ and $B, C$. then

$\left|w_{n}\right| \leq\left(A^{n-1}+\beta_{1} A^{n-3} B+\beta_{2} A^{n-5} B^{2}+\ldots+\beta_{s} B^{\left[\frac{n}{2}\right]}\right)\left|w_{1}\right|+\left(A^{n-2} B+\gamma_{1} A^{n-4} B^{2}+\right.$ $\left.\ldots+\gamma_{t} A B^{\left[\frac{n}{2}\right]}\right)\left|w_{0}\right|+\left(A^{n-2}+A^{n-3}+\ldots+1\right) C+\left(\delta_{1} A^{n-4}+\delta_{2} A^{n-5}+\ldots+\delta_{m} A+\right.$ 1) $B C+\left(\zeta_{1} A^{n-6}+\zeta_{2} A^{n-7}+\ldots+\zeta_{l} A+1\right) B^{2} C+\left(\lambda_{1} A^{n-8}+\lambda_{2} A^{n-9}+\ldots+\right.$ $\left.\lambda_{p} A+1\right) B^{3} C+\ldots, \quad n$ odd

and

$\left|w_{n}\right| \leq\left(A^{n-1}+\beta_{1} A^{n-3} B+\beta_{2} A^{n-5} B^{2}+\ldots+\beta_{s} A B^{\left[\frac{n}{2}\right]-1}\right)\left|w_{1}\right|+\left(A^{n-2} B+\right.$ $\left.\gamma_{1} A^{n-4} B^{2}+\ldots+\gamma_{t} B^{\left[\frac{n}{2}\right]}\right)\left|w_{0}\right|+\left(A^{n-2}+A^{n-3}+\ldots+1\right) C+\left(\delta_{1} A^{n-4}+\delta_{2} A^{n-5}+\right.$ $\left.\ldots+\delta_{m} A+1\right) B C+\left(\zeta_{1} A^{n-6}+\zeta_{2} A^{n-7}+\ldots+\zeta_{l} A+1\right) B^{2} C+\left(\lambda_{1} A^{n-8}+\lambda_{2} A^{n-9}+\right.$ $\left.\ldots+\lambda_{p} A+1\right) B^{3} C+\ldots, \quad n$ even

where $\beta_{s}, \gamma_{t}, \delta_{m}, \zeta_{l}, \lambda_{p}$, are constants for all $s, t, m, l$ and $p$.

The proof, using mathematical induction is straightforward.

Theorem 3.1 For arbitrary fixed $r: 0 \leq r \leq 1$, the two step approximates of Eq.(3.12) converge to the exact solution $[\underline{Y}(t, \alpha), \bar{Y}(t, \alpha)]$.

Proof. As in ordinary differential equation, it is sufficient to show :

$$
\lim _{h \rightarrow 0} \underline{y}_{N}(\alpha)=\underline{Y}(T, \alpha), \quad \lim _{h \rightarrow 0} \bar{y}_{N}(\alpha)=\bar{Y}(T, \alpha)
$$

By using exact value we get :

$$
\begin{aligned}
& \underline{Y}_{n+1}(\alpha)=\underline{Y}_{n}(\alpha)-\frac{h}{2} f\left(t_{n-1}, \bar{Y}_{n-1}(\alpha)\right)+\frac{3 h}{2} f\left(t_{n}, \underline{Y}_{n}(\alpha)\right)+\frac{5}{12} h^{3} \underline{Y}^{\prime \prime \prime}\left(\xi_{n}\right) \\
& \bar{Y}_{n+1}(\alpha)=\bar{Y}_{n}(\alpha)-\frac{h}{2} f\left(t_{n-1}, \underline{Y}_{n-1}(\alpha)\right)+\frac{3 h}{2} f\left(t_{n}, \bar{Y}_{n}(\alpha)\right)+\frac{5}{12} h^{3} \bar{Y}^{\prime \prime \prime}\left(\xi_{n}\right)
\end{aligned}
$$


where $t_{n}<\underline{\xi}_{n}, \bar{\xi}_{n}<t_{n+1}$. Consequently

$$
\begin{gathered}
\underline{Y}_{n+1}(\alpha)-\underline{y}_{n+1}(\alpha)=\underline{Y}_{n}(\alpha)-\underline{y}_{n}(\alpha)-\frac{h}{2}\left\{f\left(t_{n-1}, \bar{Y}_{n-1}(\alpha)\right)-f\left(t_{n-1}, \bar{y}_{n-1}(\alpha)\right)\right\}+ \\
\frac{3 h}{2}\left\{f\left(t_{n}, \underline{Y}_{n}(\alpha)\right)-f\left(t_{n}, \underline{y}_{n}(\alpha)\right)\right\}+\frac{5}{12} h^{3} \underline{Y}^{\prime \prime \prime}\left(\xi_{n}\right) \\
\bar{Y}_{n+1}(\alpha)-\bar{y}_{n+1}(\alpha)=\bar{Y}_{n}(\alpha)-\bar{y}_{n}(\alpha)-\frac{h}{2}\left\{f\left(t_{n-1}, \underline{Y}_{n-1}(\alpha)\right)-f\left(t_{n-1}, \underline{y}_{n-1}(\alpha)\right)\right\}+ \\
\frac{3 h}{2}\left\{f\left(t_{n}, \bar{Y}_{n}(\alpha)\right)-f\left(t_{n}, \bar{y}_{n}(\alpha)\right)\right\}+\frac{5}{12} h^{3} \bar{Y}^{\prime \prime \prime}\left(\xi_{n}\right)
\end{gathered}
$$

Denote $w_{n}=\underline{Y}_{n}(\alpha)-\underline{y}_{n}(\alpha), v_{n}=\bar{Y}_{n}(\alpha)-\bar{y}_{n}(\alpha)$. Then

$$
\begin{aligned}
&\left|w_{n+1}\right| \leq\left(1+\frac{3 h L_{1}}{2}\right)\left|w_{n}\right|+\frac{h L_{2}}{2}\left|v_{n-1}\right|+\frac{5}{12} h^{3} \underline{M} \\
&\left|v_{n+1}\right| \leq\left(1+\frac{3 h L_{3}}{2}\right)\left|v_{n}\right|+\frac{h L_{3}}{2}\left|w_{n-1}\right|+\frac{5}{12} h^{3} \bar{M}
\end{aligned}
$$

where $\underline{M}=\max _{t_{0} \leq t \leq T} \underline{Y}^{\prime \prime \prime}(t, \alpha)$ and $\bar{M}=\max _{t_{0} \leq t \leq T} \bar{Y}^{\prime \prime \prime}(t, \alpha)$ and we put $L=$ $\max \left\{L_{1}, L_{2}, L_{3}, L_{4}\right\}$ then we have :

$$
\begin{aligned}
& \left|w_{n+1}\right| \leq\left(1+\frac{3 h L}{2}\right)\left|w_{n}\right|+\frac{h L}{2}\left|v_{n-1}\right|+\frac{5}{12} h^{3} \underline{M} \\
& \left|v_{n+1}\right| \leq\left(1+\frac{3 h L}{2}\right)\left|v_{n}\right|+\frac{h L}{2}\left|w_{n-1}\right|+\frac{5}{12} h^{3} \bar{M}
\end{aligned}
$$

thus

$$
\left|w_{n+1}\right|,\left|v_{n+1}\right| \leq\left|u_{n+1}\right| \leq\left(1+\frac{3 h L}{2}\right)\left|u_{n}\right|+\frac{h L}{2}\left|u_{n-1}\right|+\frac{5}{12} h^{3}[\underline{M}+\bar{M}]
$$

where $\left|u_{n}\right|=\left|w_{n}\right|+\left|v_{n}\right|$. By lemma (3.1) and $w_{0}=v_{0}=0$ we obtain :

$\left|w_{n}\right|,\left|v_{n}\right| \leq \frac{\left(1+\frac{3 h L}{2}\right)^{n-1}-1}{\frac{3 h L}{2}} \times \frac{5}{12} h^{3}[\underline{M}+\bar{M}]+\left\{\delta_{1}\left(1+\frac{3 h L}{2}\right)^{n-4}+\delta_{2}\left(1+\frac{3 h L}{2}\right)^{n-5}+\right.$ $\left.\ldots+\delta_{m}\left(1+\frac{3 h L}{2}\right)+1\right\}\left(\frac{h L}{2}\right)^{2} \frac{5}{12} h^{3}[\underline{M}+\bar{M}]+\left\{\zeta_{1}\left(1+\frac{3 h L}{2}\right)^{n-6}+\zeta_{2}\left(1+\frac{3 h L}{2}\right)^{n-7}+\right.$ $\left.\ldots+\zeta_{l}\left(1+\frac{3 h L}{2}\right)+1\right\}\left(\frac{h L^{3}}{2}\right) \frac{5}{12} h^{3}[\underline{M}+\bar{M}]+\left\{\lambda_{1}\left(1+\frac{3 h L}{2}\right)^{n-8}+\lambda_{2}\left(1+\frac{3 h L}{2}\right)^{n-9}+\right.$ $\left.\ldots+\lambda_{p}\left(1+\frac{3 h L}{2}\right)+1\right\}\left(\frac{h L}{2}\right)^{4} \frac{5}{12} h^{3}[\underline{M}+\bar{M}]+\ldots$

therefore

$\left|w_{n}\right|,\left|v_{n}\right| \leq \frac{5 e^{\frac{3 L\left(T-t_{1}\right)}{2}}}{18 L} \times h^{2}[\underline{M}+\bar{M}]+\left\{\delta_{1}\left(1+\frac{3 h L}{2}\right)^{n-4}+\delta_{2}\left(1+\frac{3 h L}{2}\right)^{n-5}+\ldots+\right.$ $\left.\delta_{m}\left(1+\frac{3 h L}{2}\right)+1\right\}\left(\frac{h L}{2}\right)^{2} \frac{5}{12} h^{3}[\underline{M}+\bar{M}]+\left\{\zeta_{1}\left(1+\frac{3 h L}{2}\right)^{n-6}+\zeta_{2}\left(1+\frac{3 h L}{2}\right)^{n-7}+\ldots+\right.$ $\left.\zeta_{l}\left(1+\frac{3 h L}{2}\right)+1\right\}\left(\frac{h L^{3}}{2}\right) \frac{5}{12} h^{3}[\underline{M}+\bar{M}]+\left\{\lambda_{1}\left(1+\frac{3 h L}{2}\right)^{n-8}+\lambda_{2}\left(1+\frac{3 h L}{2}\right)^{n-9}+\ldots+\right.$ $\left.\lambda_{p}\left(1+\frac{3 h L}{2}\right)+1\right\}\left(\frac{h L}{2}\right)^{4} \frac{5}{12} h^{3}[\underline{M}+\bar{M}]+\ldots$

and if $h \rightarrow 0$ we get $w_{n} \rightarrow 0, v_{n} \rightarrow 0$ which concludes the proof. 


\section{4 example}

Consider the initial value problem

$$
\begin{gathered}
y^{\prime}(t)=-y(t) \\
y(0)=(0.96+0.04 \alpha, 1.01-0.01 \alpha) \\
y(0.1)=\left((0.96+0.04 \alpha) e^{-0.1},(1.01-0.01 \alpha) e^{-0.1}\right)
\end{gathered}
$$

The exact solution at $t=1$ is given by

$$
Y(1, \alpha)=\left((0.96+0.04 \alpha) e^{-1},(1.01-0.01 \alpha) e^{-1}\right), 0 \leq \alpha \leq 1 .
$$

Using the two step method with $N=10$ we obtain

\begin{tabular}{|c|c|c|c|c|c|c|}
\hline$\alpha$ & $\underline{y}$ & $\underline{Y}$ & ERROR & $\bar{y}$ & $\bar{Y}$ & ERROR \\
\hline 0 & .2527616437 & .3531642636 & -.1004026199 & .4748452781 & .3715582356 & .1032870425 \\
\hline 0.1 & .2644198408 & .3546357813 & $-0.902159405 \mathrm{e}-1$ & .4642951118 & .3711903562 & $0.931047556 \mathrm{e}-1$ \\
\hline 0.2 & .2760780380 & .3561072991 & $-0.800292611 \mathrm{e}-1$ & .4537449455 & .3708224767 & $0.829224688 \mathrm{e}-1$ \\
\hline 0.3 & .2877362351 & .3575788168 & $-0.698425817 \mathrm{e}-1$ & .4431947792 & .3704545973 & $0.727401819 \mathrm{e}-1$ \\
\hline 0.4 & .2993944323 & .3590503346 & $-0.596559023 \mathrm{e}-1$ & .4326446129 & .3700867178 & $0.625578951 \mathrm{e}-1$ \\
\hline 0.5 & .3110526294 & .3605218524 & $-0.494692230 \mathrm{e}-1$ & .4220944466 & .3697188384 & $0.523756082 \mathrm{e}-1$ \\
\hline 0.6 & .3227108265 & .3619933701 & $-0.392825436 \mathrm{e}-1$ & .4115442803 & .3693509590 & $0.421933213 \mathrm{e}-1$ \\
\hline 0.7 & .3343690237 & .3634648879 & $-0.290958642 \mathrm{e}-1$ & .4009941140 & .3689830795 & $0.320110345 \mathrm{e}-1$ \\
\hline 0.8 & .3460272208 & .3649364057 & $-0.189091849 \mathrm{e}-1$ & .3904439477 & .3686152001 & $0.218287476 \mathrm{e}-1$ \\
\hline 0.9 & .3576854180 & .3664079234 & $-0.87225054 \mathrm{e}-2$ & .3798937814 & .3682473206 & $0.116464608 \mathrm{e}-1$ \\
\hline 1 & .3693436151 & .3678794412 & $0.14641739 \mathrm{e}-2$ & .3693436151 & .3678794412 & $0.14641739 \mathrm{e}-2$ \\
\hline
\end{tabular}

\section{Conclusion}

We note that the convergence order of the Euler method in [8] is $O(h)$. It is shown that in proposed method, the convergence order is $O\left(h^{2}\right)$.

\section{References}

[1] S. Abbasbandy, T. Allahviranloo, Oscar Lopez-Pouso, Juan J. Nieto, Numerical Methods for Fuzzy Differential Inclusions, Journal of Computer and Mathematics With Applications 48 (2004) 1633-1641.

[2] S. Abbasbandy, T. Allahviranloo, Numerical Solutions of Fuzzy Differential Equations By Taylor Method, Journal of Computational Methods in Applied Mathematics 2 (2002) 113-124.

[3] S.L. Chang, L.A. Zadeh, On fuzzy mapping and control, IEEE Trans, Systems Man Cybernet. 2 (1972) 30-34. 
[4] D.Dubois, H. Prade, Towards fuzzy differential calculus: Part 3, differentiation, Fuzzy Sets and Systems 8 (1982) 225-233.

[5] O. Kaleva, Fuzzy differential equations, Fuzzy Sets and Systems 24 (1987) 301-317.

[6] O. Kaleva, The Cuachy problem for Fuzzy differential equations, Fuzzy Sets and Systems 35 (1990) 389-396.

[7] S. Seikkala, On the fuzzy initial value problem, Fuzzy Sets and Systems 24 (1987) 319-330.

[8] M. Ma, M. Friedman, A. Kandel, Numerical Solutions of fuzzy differential equations, Fuzzy Sets and Systems 105 (1999) 133-138.

[9] James J. Buckley, Thomas Feuring, Fuzzy differential equations, Fuzzy Sets and Systems 110 (2000) 43-54.

[10] William Voxman, Some remarks on distance between fuzzy numbers, Fuzzy Sets and Systems 100 (1998) 353-365.

[11] S. Seikkala, On the fuzzy initial value problem, Fuzzy sets an systems 24 (1987) 319-330.

[12] O. Kaleva, Interpolation of fuzzt data, fuzzy sets and systems, 60 (1994) 63-70.

Received: September 27, 2005 